\title{
WHERE ARE POSSIBLE WORLDS? (ARGUMENTS FOR ReALIS)*
}

\author{
GÁBOR ALBERTI-JUDIT KLEIBER \\ University of Pécs \\ Ifjúság útja 6. \\ H-7624 Pécs \\ Hungary \\ alberti.gabor@pte.hu; kleiber.judit@pte.hu
}

\begin{abstract}
We follow Pollard (2007) in assuming that the mainstream Kripke/Montagueinspired possible-worlds semantics is "a framework known to have dubious foundations" (primarily because of the granularity problem), and "worlds are constructed from propositions $[\ldots]$, and not the other way around". We intend to work out this approach in a DRT-based framework, called $\mathfrak{R}$ eALIS, in order to account for phenomena concerning referent accessibility, at the same time. We claim that our system offers a general solution to problems of intensional identity, and it is devoid of DRT's "extra level" problem - by embedding discourse representations in the world model, not directly but as parts of the representations of interpreters' minds, i.e., their (permanently changing) information states/ "internal worlds”. Hence, there is simply no intensionality in ReALIS as interpreters' "worldlets" (in description of their brains within the entire model of the universe) carry all kinds of information (BDI, guesswork, dream) typically "entrusted to" possible worlds.
\end{abstract}

Keywords: (eliminative) possible-worlds semantics, dynamic semantics, intensionality

${ }^{*}$ We are grateful to SROP-4.2.1.B-10/2/KONV/2010/KONV-2010-0002 (Developing Competitiveness of Universities in the Southern Transdanubian Region) for their contribution to our costs at SinFonIJa 4 (2011, Budapest) and ensuring the working of Research Team ReALIS for Theoretical, Computational and Cognitive Linguistics. We would also like to thank all three reviewers very much for their great efforts and numerous precious comments.

This paper is the first part of a pair of papers. The second part (AlbertiKleiber 2012) offers a review on the status of ReALIS within seminal related works in the field on the basis of the questions and comments due to the three anonymous reviewers of an earlier version of this paper. 
Our starting-point is Pollard's $(2007,33)$ conclusion pertaining to the mainstream Kripke/Montague-inspired possible-worlds semantics: "the idea of taking worlds as a primitive of semantic theory is a serious misstep". Section 1 offers a review of the most stubborn problems that the discipline of formal semantics suffers from.

We are searching a general solution in an approach based on embedding discourse representations in the world model, but not directly but as parts of the representations of interpreters' minds, i.e., their (permanently changing) information states/their "internal worlds". We call this approach ReALIS: REciprocal And Lifelong Interpretation System. In Section 2 we sketch a few relevant properties of this (S)DRT-based theory; out of which the crucial one is that there is simply no intensionality in ReALIS as interpreters' "worldlets" (in description of their brains within the entire model of the universe) carry all kinds of information (BDI, guesswork, dream) typically "entrusted to" possible worlds.

It is shown in Section 3 how Pollard's (2007) granularity problems (Hesperus/Phosphorus, omniscience) are solved-practically "disappear" - in ReALIS, due to regarding internal "images" of entities of the world as entities as well. Section 4 shows our solution to linguistic problems concerning beliefs (modal anchoring, Hob/Nob sentences). ${ }^{1}$

\section{Dubious foundations of (post-)Montagovian formal systems}

\subsection{Granularity problems}

Our starting point is Pollard's (2007) criticism on the mainstream Kripke/ Montague-inspired possible-worlds semantics.

He calls it "a framework known to have dubious foundations" (op.cit., 1) primarily because of the granularity problem, illustrated in (1) below, and he is led to the conclusion that "the idea of taking worlds as a

${ }^{1}$ Alberti-Kleiber (2012) offers a systematic review of phenomena concerning identity/identification of entities (e.g. that of actors and stunt men, that of Theseus' ships). To meet the requirements of all three anonymous reviewers of the first version of this paper, we have devoted the major part of the second article to the comparison of ReALIS with seminal related works in the field: Landman's (1986) theory on partial objects and DRT (Kamp et al. 2011), for instance. We also suggest that $\mathfrak{R e A L I S}$, due to its mind representation, can be regarded as a formal cognitive system (e.g., Schnell 2006). 
primitive of semantic theory is a serious misstep" (op.cit., 33). Even the seminal book of teaching Montague Grammar (Dowty et al. 1981, 124) admits these "dubious foundations" in the course of discussing the problem of necessity and possibility: "Would this be an enlightening way of analyzing the semantics of necessity and possibility? Many philosophers of language have unequivocally answered "no" to this question; they have contended that since "possible worlds" are surely vague and ill-understood entities [...], it cannot help to explain one mysterious semantic concept (necessity) in terms of an even more mysterious one (possible worlds)" (italicized by the authors).

(1) Types of the granularity problem:

(a) The ancients realized that [Hesperus was Hesperus $]_{\mathrm{S} 1} /[\text { Hesperus was Phosphorus }]_{\mathrm{S} 2}$.

(b) Jim knows $[\text { Phil is a woodchuck }]_{\mathrm{S} 1} /[\text { Phil is a groundhog }]_{\mathrm{S} 2}$.

(c) Problem of omniscience: Paris Hilton knows that [10 is greater than 7$]_{\mathrm{S} 1} /[\text { there are infinitely many (twin) prime numbers }]_{\mathrm{S} 2}(\mathrm{~S} 3)$.

(d) A conversation overheard in ancient Babylonia: "Look, there is Hesperus, brighter than it was yesterday." "Phosphorus was also bright this morning."

"Really? It wasn't that bright yesterday."

Hesperus and Phosphorus in (1a) ( $\rightarrow$ Venus), woodchuck and groundhog in (1b) ( $\rightarrow$ the same marmot-like species), and the three constantly true sentence variants in (1c) have (pairwise) the same referential content whilst $(1 \mathrm{a} / \mathrm{S} 1)$ and $(1 \mathrm{c} / \mathrm{S} 1)$ are trivially true in contrast to the contingent statements (1a/S2), (1c/S2), (1c/S3) ${ }^{2}$ and the also contingent ones (1b/S1) and $(1 \mathrm{~b} / \mathrm{S} 2)$ which may potentially take distinct truth values.

What this comparison between (natural human intuition on) meanings of these sentences shows, can be summarized as follows: having the same reference/meaning is not a sufficient condition to allow replacement of one name/sentence for another in a larger expression while preserving truth - that is what Pollard calls the Granulatity Problem and qualifies

2 To be more precise, the theorem pertaining to twin prime numbers $(1 \mathrm{c} / \mathrm{S} 3)$ is only a conjecture that has not been proved so far (a few examples of twin prime numbers: $\langle 3,5\rangle,\langle 5,7\rangle,\langle 11,13\rangle,\langle 17,19\rangle,\langle 29,31\rangle,\langle 41,43\rangle)$. If it is true, nevertheless, then it is "as true as" the fact that 10 is greater than 7 ; whilst the sets of those knowing S1/S2/S3 are three radically different sets (cca. everybody/those interested in mathematics/nobody). 
as the critical problem that the mainstream Kripke/Montague-inspired possible-worlds semantics suffers from.

Landman (1986, 97) discusses another aspect of the HesperusPhosphorus problem, the problem of "intersubjective agreement", illustrated in (1d) above. He argues,

"in using the word 'Hesperus' both persons assume that they talk about the same object. [...] How come, they are at the same time so sure that they talk about the same things, and not each of them about their own private experiences, and still are not aware that the things they talk about are actually one and the same object?"

He formulates the essence of the problem that he calls "the main problem in semantics since Frege" as follows:

\begin{abstract}
"Where are the objects we talk about? If they are in our minds, then the above conversation involves four objects, two for each language user; if they are in the world, then it involves only one object, the planet Venus. But if we ask the Babylonians themselves, the two would not disagree about the number of objects: there are two: Hesperus and Phosphorus. [...] The task of semantics is to find the proper place for this intersubjective agreement [2 objects], in between subjectivity [4 objects] and objectivity [1 object]."
\end{abstract}

\title{
1.2. Problem of the compositionality of reference
}

Another problem mentioned by Pollard (2007, 30-1; see also his footnote 36) lies in "the standard view, [viz.] reference is compositional".

(2) The problem of using the customary sense as the reference:

$\left[\left(\text { Justin Timberlake knows that) }\left[\text { Paris Hilton believes }[\text { snow is white }]_{\mathrm{S} 3}\right]_{\mathrm{S} 2}\right]_{\mathrm{S} 1}\right.$.

That is why, claims Pollard, "Frege had to resort to claiming that utterances of sentences in certain contexts [e.g. S3 in (2)] had the customary sense as the reference," which requires "sleight of hand".

\subsection{Problems of accessibility of referents}

A third stubborn problem of the mainstream post-Montagovian formal semantics, which is the starting point of DRT (Kamp et al. 2011), concerns distinct accessibility of certain referents $(3 \mathrm{a}-\mathrm{b})$ in logically equivalent sentences $(3 \mathrm{c})$ : 
(3) Problem of referent accessibility and DRT's solution:

(a) [[A delegate arrived. $]_{\mathrm{S} 1}$ She registered. $]_{\text {Discourse1 }}$

(b) [[It is not the case that every delegate failed to arrive. $]_{\mathrm{S} 2}$ $*$ She registered. $]_{\text {Discourse } 2}$

(c) The formulae attributed to S1 and S2 are logically equivalent: $\exists x \Phi \Leftrightarrow \neg \forall x \neg P h i$.

(d) The representation of Discourse1/Discourse2 in DRT: "Enclosed", $x$ is not accessible to $y$.
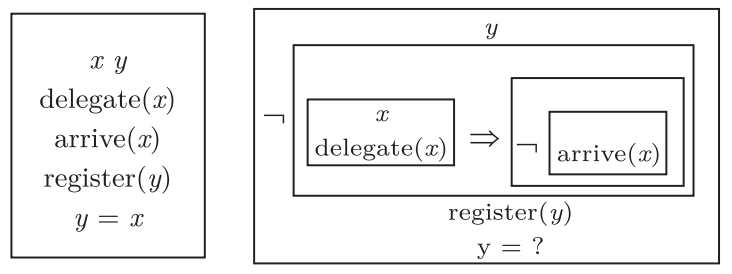

DRT's solution relies on placing referents belonging to pronouns and (other) definite and indefinite nominal expressions in a hierarchical "box structure" calculated on the basis of sentence and discourse structure. The elementary box structure above to the left corresponds to the simpler discourse (3a), with referent $x$ belonging to a delegate and $y$ belonging to she. As referents $x$ and $y$ can be found in the same "box", $x$ is defined to be accessible to $y$-which accounts for the successful coreference between the pronoun and the indefinite nominal expression. In the complex box structure, however, which corresponds to the logically complex discourse in (3b), the embedded boxes (whose subordinate status is due to negation and universal quantification) practically "hide" referent $x$ from referent $y$ - which accounts for the failure of coreference between the pronoun and the indefinite nominal expression.

\subsection{The problem of "extra level"}

DRT's solution comes with the cost of introducing an extra level of representation, that of discourse (representation) structures (3d), i.e., DRSs.

Those insisting on Montague's "heritage" (e.g., Groenendijk-Stokhof 1991) qualify DRSs as an illegitimate extra level of representation between syntactic structure and the model of world in the course of interpretation, which can - and necessarily should - be eliminated. 


\title{
1.5. Problems of intensional identity
}

This subsection is devoted to the demonstration of two (related) problems concerning intensional identity, which often occur in the literature of formal semantics.

(4) The Hob-Nob problem and the puzzle of modal anchoring:

(a) Hob believes that a witch has killed Cob's cow and Nob thinks that she has blighted Bob's sow.

(b) Mary thought that there was a castle behind the trees. The castle turned out to be a huge oak tree.

The "Hob-Nob Problem" is sketched by Kamp et al. $(2011,5.4)$ as follows:

\begin{abstract}
"Geach [1962] pointed out that this sentence [(4a)] could be used truthfully in a report composed by a journalist describing the goings-on in some remote rural backwater, even if the journalist herself is persuaded that witches do not exist. This is a problem for the application of standard logical notation to the representation of truth-conditional content. For in order that the pronoun she in the belief attribution to Nob be bound by the "existential quantifier" a witch in the belief attribution to Hob, this quantifier would have to take scope over the two belief attributions. But this would, on the standard interpretation of quantification theory, imply that there are witches in the world in which Hob, Nob and the journalist live. That is something to which the journalist would under no conditions want to commit herself. And it is something to which ... [(4a)] does not commit her."
\end{abstract}

Following Roberts (1996b, 237), we can add that "the problem of intensional identity in [Hob-Nob examples (4a)] ... reduces to the more general problem of intensional identity, i.e. identity across possible worlds. Such an approach, of course, encounters difficult problems in attempting to specify what it is for two individuals to believe in the existence of the same, possibly mythical entity (see Lewis (1986) and the references therein)."

The similar puzzle in the two-sentence text (4b) is that "a noun phrase [the castle] is modally subordinated to a constituent occurring in previous discourse, while the sentence the noun phrase is part of is not modally subordinated" (Farkas 1993, quoted by Roberts 1996a, 243). This contradictory case means a serious problem to semantic theories based on the elimination of possible worlds, as different parts of the sentence in question require distinct ways of eliminating possible worlds. 


\section{Foundations of $\Re$ eALIS}

We follow Pollard $(2007,34)$ in assuming that "worlds are constructed from propositions [...], and not the other way around" (1.1-2), but intend to work out this approach in a DRT-based framework, called $\mathfrak{R}$ eALIS, in order to account for phenomena concerning referent accessibility (1.3), at the same time. We claim, however, that our system is devoid of DRT's "extra level" problem (1.4) and offers a general solution to problems of intensional identity (1.5).

ReALIS, REciprocal And Lifelong Interpretation System, can be introduced as a new "post-Montagovian" theory (Dowty et al. 1981) concerning the formal interpretation of sentences constituting coherent discourses (Kamp et al. 2011), with a lifelong model of lexical, interpersonal and cultural/encyclopedic knowledge of interpreters in its center including their reciprocal knowledge on each other. In what follows we sketch a few relevant properties of ReALIS. We also offer the interested reader our new publications in Hungarian (see Alberti 2011a;b and further references therein), and the 40-page-long definition system in English (Alberti 2009: http://lingua.btk.pte.hu/realispapers).

What is relevant here is that in our approach, DRSs - gigantic ones, of course - are used as lifelong representations of interpreters' information states (Alberti 2000), and what serve as ilks that play the role of possible worlds are embedded DRS boxes, which are practically finite information pools not closed under logical operations. Due to unbounded embedding of "boxes", we can express interpreters' beliefs/desires/intentions including BDI's concerning BDI's (concerning BDI's)* of each other. An interpreter's information state, thus, is captured formally as a labeled tree system of "worldlets" (the above mentioned finite information pools) and can be construed practically as the description of his/her brain-his/her "internal world", which is a part of the entire world model also containing the external world.

Hence, ReALIS requires no extra level of representation (1.4), as discourse representations, in company with representations of other contents of the above mentioned internal worlds, are not outside the world model - see the comparison in (5) below.

In order to understand all details, we should return to Montague's heritage mentioned in 1.4. He himself also applied a level of representation between syntactic structure and world model: a level of logical formulae, about which he proved that theoretically this level can be elim- 
inated (Dowty et al. 1981). Linguistic problems like the one sketched in 1.3, however, seem to have proven the opposite, some principle of "discourse representationalism". Dekker (2000), a researcher belonging to Groenendijk and Stokhof's antirepresentationalist Amsterdam Circle in the nineties, tends to accept in 2000 that "some [intermediate] level of representation is indispensable in modeling the interpretation of natural language".

The thesis of $\Re$ eALIS in this context, then, can be formulated as follows: By embedding discourse representations in the world model, we can get rid of the intermediate level of representation while preserving its content and relevant structural characteristics; and this idea can be carried out in the larger-scale framework of embedding discourse representations in the world model not directly but as parts of the representations of interpreters' minds, i.e., their (permanently changing) information states, that is, their internal worlds. We argue that in this way representationalists' useful referent hierarchies (1.3) are saved while antirepresentationalists' principal theoretical demand pertaining to the elimination of the extra level of representation has also been satisfied.

It might be raised that in the $\Re$ eALIS approach a double nature is attributed to human minds, which might cause a critical meta-theoretical difficulty: they are representations about the "world outside", on the one hand, but - as parts of the entire world model - they are to be represented as well, on the other.

This is no theoretical problem, however, if we apply a simultaneously recursive definition of "the whole": the world "outside" and all interpreters' permanently changing information states (Alberti 2011a, http://lingua.btk.pte.hu/realispapers).

$\Re$ ReALIS, thus, can be formally defined by simultaneous recursion as an epistemic multi-agent system $\mathfrak{R}=\left\langle\mathrm{W}_{\mathrm{o}}, \mathrm{W}, \mathrm{Dyn}, \mathrm{Tru}\right\rangle$ (Fagin et al. 1995) where "agents" get information about the world around them (including each other's brains). $\mathrm{W}_{\text {o }}$ denotes the external world, a "full history", on the basis of which both static (truth-conditional) evaluation (Tru) and dynamic (DRS-constructing) interpretation (Dyn) can be carried out, in cooperation with each other (Kamp et al. 2011). W is a function where $\mathrm{W}[i, t]$ is interpreter $i$ 's information state (=internal world) at moment $t$. W $[i, t]$ is a labeled tree system of worldlets, as has been mentioned. The interpretation of modal sentences can be based on certain worldlets instead of the external world W. 
What this means is no less than there is simply no intensionality in ReALIS as interpreters' worldlets (in description of their brains within the entire model of the universe) carry all kinds of information (BDI, guesswork, dream) typically "entrusted to" possible worlds. In other words, interpretation is always extensional, with its basis either $\mathrm{W}_{\mathrm{o}}$ or a sector of an $i$ 's $\mathrm{W}[i, t]$, or, quite frequently, some combination of the external world and one or more interpreters' different worldlets.

This approach can also be formulated as a hypothesis: all (linguistic) problems whose solution is held to require possible worlds can be solved by means of worldlets.

What kinds of problems should this model face? Numerous problems have appeared and further ones may appear in the course of precisely defining ReALIS - but nothing has seemed to ultimately block the endeavor as a whole. The analyses in the following sections of the most stubborn problems in the area are intended to persuade the reader.

\section{How do granularity problems disappear in ReALIS?}

\subsection{Stars and twins: Anchoring internal entities to external ones and to each other}

A critical difference between the approach of ReALIS and that of DRT lies in the choice of basic objects of the theoretical model: in DRT every concept relies on (the "box structure" of) DRSs whilst in ReALIS it is the universe of (external and internal) entities that a multiply complex network of relations is based upon.

In $\mathfrak{R e A L I S}$ each instance of observation of an external entity by an interpreter comes with producing a new internal entity, a referent, in his/her internal world. Identifying referents in this approach is anchoring one to another $\left(r_{k} \rightarrow r_{l}\right)$ by a relation $\alpha$, which requires a step of accommodation considered to depend on the interpreter's decision chiefly on the basis of pragmatic facts.

Let us turn to the problem of Evening/Morning Star, whose illustration is repeated in (5) below. What is decisive here is that external identity provides a natural basis for internal identification, of course - what can be regarded as an instance of perfect observation; but internal identification ultimately depends on the interpreter and nobody else. The figure in $\left(6^{\prime}\right)$ serves as an illustration of this dependence. 
(5) Partial observation of external entities:

$\rightarrow(1 \mathrm{a})$ The ancients realized that

[Hesperus was Hesperus $]_{\mathrm{S} 1} /[\text { Hesperus was Phosphorus }]_{\mathrm{S} 2}$.

(6) False observation of external entities:

Mary believes that

[the married Peter Caruthers is the same person as the bachelor Paul Caruthers] $\mathrm{S} 1$ / [who kissed Sue on Monday is the same person as who kissed Doris on Friday $]_{\text {S2 }}$.

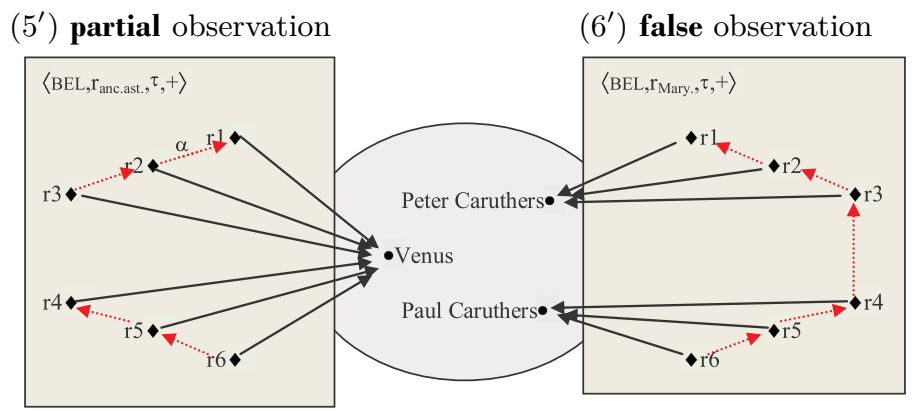

Referents $r 1, r 2, r 3$ in $\left(6^{\prime}\right)$ are intended to register observations, at a sequence of moments, of Venus in the evening sky by an ancient astronomer; and $r 4, r 5, r 6$ are observations of Venus in the morning sky. Referent $r 2$ is easy to anchor to $r 1$, and $r 3$ is also easy to anchor to r2: they are observed in similar positions in the evening sky. Referents $r 4, r 5$ and $r 6$ are also easy to anchor to each other. Anchoring $r 4$ to $r 3$, however, requires profound knowledge of astronomy; accommodating the piece of information concerning the identity of $r_{4}$ and $r 3$, hence, is an accommodation that can be supposed to be such that certain interpreters will perform while others will not. Interpreters belonging to the latter group can be said to get partial observation (Alberti 2009, 2.1.4.5/2011a, 4.1.4.5) of the external entity Venus. The solution relies on the assumption that interpreters' internal worlds consist of logically not closed, finite pools of information, referred to in section $\mathbf{2}$ as worldlets.

The same style of solution is at hand for the problem of indistinguishable twins, illustrated in (6) above. Thus Peter and Paul are supposed to be indistinguishable twin brothers. Nevertheless, if Mary is a rational person, the version of $(6 / \mathrm{S} 1)$ is strange: to believe in the identity of two persons with different names and different marital statuses, whilst version (6/S2) is completely all right under appropriate conditions. 
Let us consider the figure in $\left(6^{\prime}\right)$ above. Referents $r 1, r 2$ and $r 3$ in Mary's internal world are supposed to have come from observations of Peter while the other three referents, also belonging to Mary's universe of internal entities, correspond to his twin brother Paul. Here anchoring of r4 to $r 3$ is an instance of accommodation which Mary is likely to perform if she does not know, for instance, that his colleague Peter has a brother at all. Performing this accommodation depends on Mary alone and it is a rational operation on the basis of the brothers' exterior similarity. What has been discussed here can be called a case of false observation: in the interpreter's information state has appeared a class of identified referents belonging to different entities outside.

This solution also relies on the assumption that interpreters' internal worlds consist of logically not closed, finite worldlets. In the ideal, or default, case, obviously, worldlets are true reflections of a finite segment of the external world, in the precise sense that they show an isomorphic picture of the entity structure outside. Creating this internal picture typically requires trivial steps of accommodation; but do require accommodation, so potentially there might appear cases when a necessary step of accommodation will not be performed or a wrong step of accommodation will be performed. The famous semantic problems capture these very cases.

\subsection{Equivalent statements in internal worlds}

This subsection is devoted to the problem of equivalent statements in modal contexts, mentioned in (1b-c) as a special case of what Pollard (2007) calls the Granularity Problem. Our solution relies also on the approach that internal worldlets reflecting the external world are finite pools of information not (automatically) closed under logical operations in contrast to possible worlds.

In ReALIS, thus, logical operations may take place as accommodations in appropriate worldlets, interpreter-dependently to a certain extent, and not automatically. Worldlets may be regarded as constructions "converging into", but never arriving at, logically closed, infinite possible-world constructions.

Suppose, for instance, Mary knows all the axioms on the basis of which theorems $e 1$, e2 and $e^{3}$ in $(7 \mathrm{a}-\mathrm{c})$ below can be proved. If theorem $e 3$ is true (see fn. 3), then the three theorems are equivalently true in every "rational" possible world (which are based upon the customary 
mathematical axioms) while the three modal sentences in $(7 \mathrm{a}-\mathrm{c})$ are not equivalent at all: (7a) is obviously true, (7c) is obviously false, and (7b) is dependent on Mary's knowledge on maths. Figure (7h) shows how this radical difference can be accounted for in the approach offered by the model of $\Re$ eALIS.

(7) Incontingent truths:

(a) Mary knows that $[10 \text { is greater than } 7]_{\mathrm{e} 1} \cdot \rightarrow(1 \mathrm{c})$

(b) Mary knows that [there are infinitely many prime numbers $]_{\mathrm{e} 2} \cdot \rightarrow(1 \mathrm{c})$

(c) Mary knows that [there are infinitely many twin prime numbers $]_{\mathrm{e} 3} \cdot \rightarrow$ (1c)

(d-f) We need a prime number greater than $200 \ldots$
(d) 210 may be a good choice.
(e4: "210 is a prime number.")
(e) 221 may be a good choice.
(e5: "221 is a prime number.")
(f) 211 may be a good choice.
(e6: "211 is a prime number.")

(g) Jim knows $[\text { Phil is a woodchuck }]_{\mathrm{S} 1} /[\text { Phil is a groundhog }]_{\mathrm{S} 2} \cdot \rightarrow(1 \mathrm{~b})$

(h)

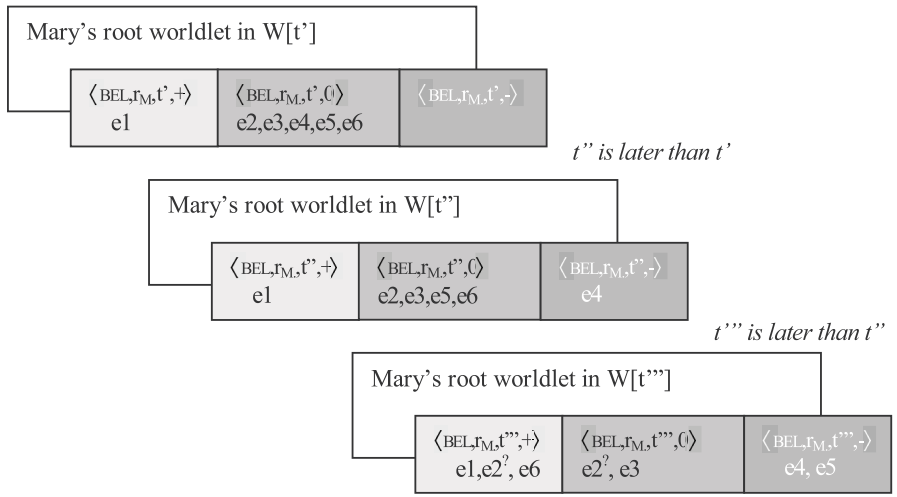

What corresponds to the well-known "box structure" of DRT is the labeled partial ordering of worldlets in ReALIS (Alberti 2009, 1.2.4/2011a, 3.2.4). We would like to demonstrate these labels by means of the representations in $(7 \mathrm{~h})$. They are quadruples providing the following factors of a label: its modality (belief/desire/intention/supposition/etc.), its direct host, its moment and its polarity (positive/neutral/negative). What is described by the upper box triplet in $(7 \mathrm{~h})$, then, is that at a moment $t^{\prime}$ Mary $\left(r_{\mathrm{M}}\right)$ strongly "believes in" (the trivial) e1 while she has not decided if $e 2$ and $e 3$ are true or false - independently of what is real in the external world. Theorem $e$ 2, for instance, is true in the external world, and Mary may be assumed to have reached an information state, at a 
moment $t^{\prime \prime \prime}$, in which $e^{2}$ has already got in the positive worldlet sector, which corresponds to the external fact that $e^{2}$ is true. This change requires much thinking (or learning), which can be regarded as a form of accommodation, again, in our approach. We can also measure the amount of accommodation by assuming that $e^{2}$ appears in the positive sector of a worldlet responsible for someone's knowledge not so soon as $t^{\prime \prime}$ but only later (at $t^{\prime \prime \prime}$ ), and $e 3$ will never enter a positive sector like this.

We show another problem in order to demonstrate this toolbox of ReALIS convincingly. Let us compare the three two-sentence discourses in $(7 \mathrm{~d}-\mathrm{f})$. On the one hand, $(7 \mathrm{e}-\mathrm{f})$ are reasonable statements in contrast to $(7 \mathrm{~d})$, as 210 is obviously no prime number. On the other hand, however, $(7 \mathrm{~d}-\mathrm{e})$ are in opposition to $(7 \mathrm{f})$, because it is only the number mentioned in (7f) is a prime number as $221=13 \cdot 17$ in (7e).

In the approach due to ReALIS, both sides can be accounted for. The external truth concerning the numbers in question is captured by the final three-sector worldlet at moment $t^{\prime \prime \prime}$ in (7h) above: eventualities $e 4, e 5$ and $e 6$ are distributed according to this external truth, expressing that Mary can reach an information state truly reflecting the facts that only 211 is a prime number. The series of information states, however, expresses that 211 and 221 are similar in the respect that they are not easy to decide if they belong to the class of primes. This decision ("removing" $e 5$ and $e 6$ from a neutral worldlet of belief into one of $+/-$ polarity) requires much more accommodation from the interpreter than that concerning $e 4$.

\subsection{Synonyms in modal contexts}

It is worth devoting a separate subsection to the problem of synonyms, whose illustration was repeated in $(7 \mathrm{~g})$ above, with respect to its connection to the general question of lexical acquisition.

In the light of what has been said so far our proposal is straightforward: despite that woodchucks and groundhogs are the same in the external world, it is only Jim's internal world $\mathrm{W}\left[r_{\mathrm{Jim}}, t\right]$ that counts. Hence, the two sentences in $(7 \mathrm{~g})$ above may be assigned different truth values, depending on the finite content of Jim's corresponding worldlets of knowledge.

One might think that this solution is suspiciously simple; and what ReALIS will not be capable of capturing is the prototypical case in which sentences containing synonymous words are evaluated alike even in modal contexts like the one shown in $(7 \mathrm{~g})$. 
This is not the case, fortunately. Our toolbox is straightforwardly suitable for formally indicating the anomalous nature of the relevant sector of Jim's internal world. What can be defined as the ideal case is when worldlets of knowledge of interpreters truly reflect corresponding sectors of the external world. It would threaten the "survival" of a community of speakers if the "anomalous situation" occurred too frequently. That is why the content of worldlets of knowledge is to be hypothesized to "converge into" a state of more and more truly mapping the external world. This approach practically provides a theory concerning language acquisition, i.e., children's acquisition of lexical meanings as these meanings have been conventionalized in their parents' community - remaining a certain amount of possibility open for "mutation".

\section{The extensional treatment of beliefs in ReALIS}

\subsection{Truth-conditional evaluation on the basis of the content of human brains}

First of all, let us return to the problem of compositionality of reference (1.2). As a preparation, we should sketch how the two kinds of interpretation (Kamp et al. 2011) are captured in ReALIS.

Dynamic interpretation of a sentence (or rather, discourse) is defined as extending the interpreter's information state (Alberti 2009, 2.2/2011, $4.2)$. What practically takes place in the course of this extension is that new sectors are built up in the interpreter's internal world due to the (morpheme by morpheme) consumption of the input performance: there will appear new blocks of partially ordered labeled worldlets.

The ideal interpreter, for instance, will construct the worldlet system shown in (8b) below as (the relevant part of) the dynamic interpretation of $(8 \mathrm{a})=(2)$ (and then (s)he is to embed it in his/her earlier internal world by anchoring this "floating" block of worldlets to the "activated" point(s) of the information state; where this activation is controlled by anaphoric and presupposition-carrying expressions). The information (8b) expresses is as follows from worldlet to worldlet: The interpreter attributes a piece of knowledge ("maximal" belief) to Justin Timberlake (e1), which pertains to a belief of Paris Hilton (e2). This belief (e2) pertains to a proposition concerning snow according to which the supposition that something (referent r3) is of snow (e3) typically implies that it's white (e4). 
Static interpretation (truth evaluation) of a sentence is defined on the basis of the external world $\mathrm{W}_{\mathrm{o}}$ or/and the content of certain worldlets of potentially more interpreters (Alberti 2009, 2.3/2011, 4.3). It is the union of these structures $\left.\left(\mathrm{W}_{\mathrm{o}}+\mathrm{W}[i, t]\right)\right)$ that the structure of the representation which is the output of dynamic interpretation $(\mathrm{W}[i, t])$ is to be compared with: whether a sufficient pattern matching can be pointed out.

Now the truth of $(8 \mathrm{a})$, partly depending on the precise interpretation of the English verbs know and believe, is primarily determined by the content of two internal worlds, those of Justin Timberlake and Paris Hilton. (8a) is true if the worldlet quadruplet in (8b) is isomorphic to a sector of JT's internal world ("he does know S2") and the worldlet triplet in (8c) is isomorphic to a sector of PH's internal world ("she does believe S3"). This 'isomorphism' is a kind of pattern matching, like traditional interpretation, which is essentially pattern matching, too, between some representation of sentence structure and some model of the external world.

What makes the former pattern matching possible in ReALIS, is that the output of dynamic interpretation (genuinely a representation) will simultaneously serve as a contribution to the world model.

(8) How to represent and interpret BDI?

(a) $\left[\left(J u s t i n \text { Timberlake knows that) }[\text { Paris Hilton believes [snow is white }]_{\mathrm{S} 3}\right]_{\mathrm{S} 2}\right]_{\mathrm{S} 1}$. $\rightarrow(2)$

(b)

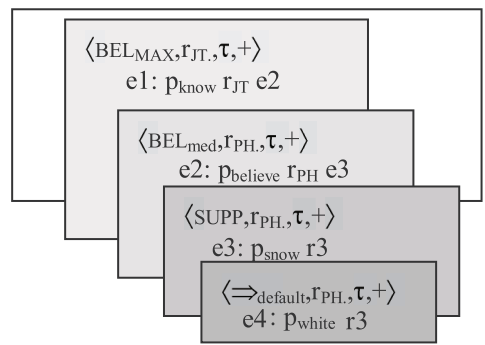

(c)

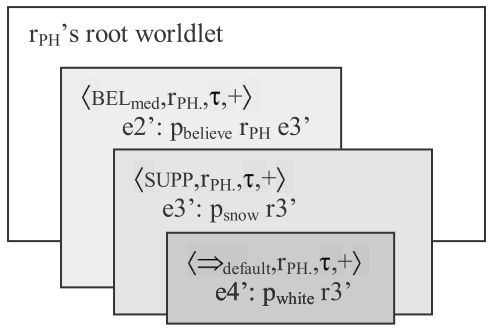

\subsection{Modal anchoring}

As was pointed out in 1.5, cross-reference between elements in distinct modal contexts, i.e., modal anchoring, means a serious problem to semantic theories based on the elimination of possible worlds, as different parts of the sentence/discourse in question require distinct ways of eliminating possible worlds. What correspond to these "distinct modal contexts" in 
$\Re$ ReALIS, however, belong to the same world model (as all internal worlds belong to the same, single, world model); hence, their referents can be anchored to each other (under appropriate conditions).

The representation in (9a), for instance, shows the relevant part of an ideal interpreter's dynamic interpretation. The contribution of the first sentence is a referent $r^{\prime}$ with the piece of information that "Mary believes that she has seen that it is a castle". The second sentence contains a singular definite description, the castle. This sentence relies on the speaker's perspective, and not Mary's one; nevertheless, the retrieval of the antecedent is successful. How is this possible?

Unicity is the relevant factor: there must be a worldlet containing a referent that is unique in that worldlet in the respect that the content of the singular definite description holds true of it. The second sentence of the two-sentence discourse in (9b) below, for instance, does not meet this requirement, and the discourse is ill-formed, indeed, while there is no change in modal context.

The precise solution of the problem in (9a) even requires some accommodation because a referent should be accessible to the other referent (1.3) to which we would like to anchor it in order to express their referential identity. Accessibility in ReALIS can be defined on the basis of worldlet hierarchy - in the most straightforward way: $r 1$ is accessible to r2 if the worldlet of $r 1$ is lower than that of $r 2$ according to the partial ordering that makes the worldlet hierarchy.

The piece of information that should be accommodated here, as a result of the singular definite expression in the second sentence of (9a), is that the speaker accepts that "there is a huge entity behind the trees, indeed". The interpreter of the discourse, thus, introduces a referent $r$ to the relative root worldlet belonging to the dynamic interpretation of the discourse. This referent $r$, then, is accessible to both $r^{\prime}$ (what Mary thought to be a castle) and $r^{\prime \prime}$ (the huge oak tree the speaker can see).

One might think that it is suspiciously simple to have recourse to accommodation again and again. We argue, however, that it a straightforward strategy of speakers that they tend to speak as little as possible and, instead, they tend to entrust as much as (they hold) possible to the interpreter's information state. Instead of attempting to ignore information not expressed explicitly by words in formal semantic analyses, we should strive for capturing this implicit information formally. The "lifelong" approach of $\mathfrak{R e A L I S}$ makes it possible, at least, to start this endeavor. 
Kálmán's (1990) example in $(9 \mathrm{c})+\left(9 \mathrm{c}^{\prime} / \mathrm{c}^{\prime \prime}\right)$ below is an excellent illustration of accommodation. In our culture a minister is a potential "distinguished participant" of a marriage whilst nothing similar holds true of a dog. Nevertheless, it is not excluded that an interpreter considers discourse $(9 \mathrm{c})+\left(9 \mathrm{c}^{\prime \prime}\right)$ to be felicitous: what is needed is, say, a piece of interpersonal knowledge about a salient dog in Peter's life. It is relevant that neither the minister in the former case, nor the dog in the latter case can be found in any kind of closure of the interpreter's information state under logical entailment, so the cohesion holds between the present sentences and ones learned long ago by the interpreter, in an unbounded chronological distance. It is not logically closed possible worlds, thus, which can provide an explanation, but the lifelong perspective offered by $\Re$ ReALIS. The singular definite expression in $\left(9 \mathrm{c}^{\prime} / \mathrm{c}^{\prime \prime}\right)$ triggers a procedure in the course of which the interpreter tries to extend his/her information state resulting from understanding the first sentence $(9 \mathrm{c})$ so that the extended information state contain a worldlet with a unique minister/ dog. This task can be executed, in the former case, by accommodating cultural information concerning marriage in our Western culture, and it might be executed, in the latter case, by accommodating interpersonal information concerning Peter.

(9) Modal anchoring as an extreme case of intensional identity

(a) Mary thought that there was a castle behind the trees. The castle turned out to be a huge oak tree. $\rightarrow(4 \mathrm{~b})$

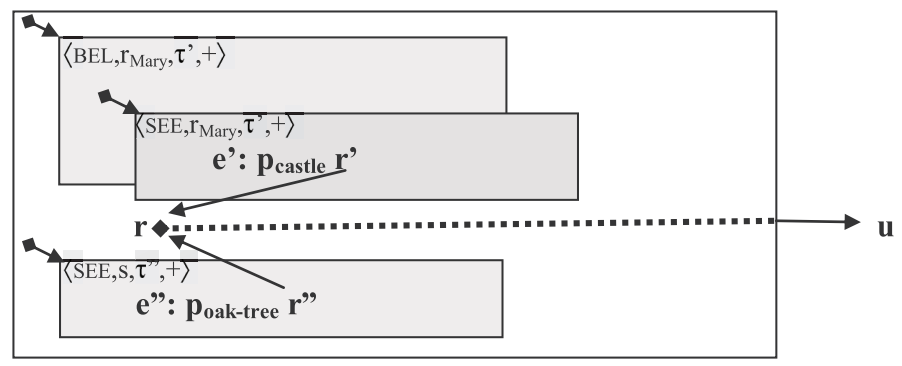

(b) Yesterday we visited two castles in an old town. * The castle was beautiful.

(c) Peter got married yesterday.

$\left(\mathrm{c}^{\prime}\right)$ The minister spoke very harshly. / ( $\left.\mathrm{c}^{\prime \prime}\right)$ ?? The dog barked very loudly.

(d) Successful reference by means of a false piece of information:

A man arrives at a motel in the company of a girl who is not his wife at all in a country where the porter (who knows the girl well...) ought to prevent them, lawfully, to share the same room. This is against his financial interest, 
however. Hence, the girl in question will be referred to as the guest's wife by both the guest himself and the porter in spite of the fact that neither of them thinks this "presupposition" to be true and, moreover, neither thinks that the other considers it to be true either. The porter says: I hope your wife will enjoy this champagne.

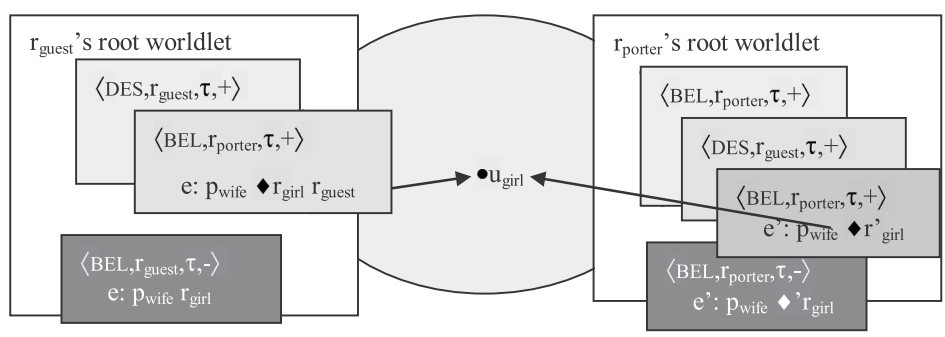

(e) Yesterday Mary met an alleged spy.

In (9d) we have sketched another context in which a piece of information ("your wife") is used to identify a person, who belongs to different modal contexts to the speaker and to the interpreter and, what is more, is held to be false by both. ReALIS offers an explanation based on unicity of the appropriate referents in certain worldlets. What the worldlet blocks above in (9d) represent is that the wife to the guest is "the unique person in the context such that he wants the porter to believe that she is his wife", and the wife to the porter is "the unique person in the context such that he thinks that the guest wants him to believe that she is his (the guest's) wife despite that he knows well that she is not the guest's wife".

A similar problem is illustrated in (9e) above. Alleged is qualified as an irregular adjective by Kiefer $(2000,188)$ on the basis of its anomalous properties compared to regular adjectives like old. We illustrate here the test of two of the anomalous properties: an alleged spy is not (necessarily) a spy whereas an old spy is a spy; and it cannot be said that He is alleged, whereas $\mathrm{He}$ is old is a well-formed sentence.

The straightforward solution in our approach is that the difference between regular and irregular adjectives is that the discourse-semantic representation of a regular adjective is a predicate (like $p_{\text {castle }}$ and $p_{\text {wife }}$ above in $(9 \mathrm{a}-\mathrm{d}))$ whilst the contribution of alleged concerns the modal label of a worldlet. The speaker refers to a person by a piece of information to whose truth she does not commit herself. She can identify him, nevertheless, by expressing that he is "a person such that some people believe that he is a spy". In this way we could account for both the fact 
that He is a spy is no correct implication (as the speaker has not committed herself to the truth of this statement) and the fact that He is alleged is ill-formed (alleged is not a predicate but a modal label).

\subsection{Nob's witch, which is Hob's witch, and Phosphorus, which is Hesperus}

Now let us turn to the Hob-Nob Problem sketched in 1.5, which illustrates that identity across possible worlds is not easy to represent formally in possible-worlds semantics in the case of beings not present in the model of the real world.

As is shown in (10b) below, ReALIS should cope with a similar problem of identification. What is explicitly expressed by the words of the famous sentence in (10a) can be represented by means of the "floating" worldlet boxes in (10b): Hob believes e1 expressing that a witch, denoted by $r_{\text {witch }}^{\prime}$, has killed Cob's cow, and Nob believes e2 expressing that a definite woman, denoted by $r^{\prime \prime}$, has blighted Bob's sow. These two referents are not accessible to each other because they can be found in incommensurate worldlets (one expressing Hob's belief and another expressing Nob's belief) in the partial ordering of the worldlet hierarchy. Hence, at the moment we cannot account for the anaphoric character of she.

(10) The Hob-Nob problem:

(a) Hob believes that a witch has killed Cob's cow and

Nob thinks that she has blighted Bob's sow. $\rightarrow(4 \mathrm{a})$

(b) A preparatory phase of dynamic interpretation:

\begin{tabular}{|c|}
\hline $\begin{array}{l}\langle ? ? ?\rangle \\
\quad \mathbf{s}_{1}: \mathbf{p}_{\text {believe }} \mathbf{r}_{\mathrm{Hob}} \mathbf{e}_{1}\end{array}$ \\
\hline \begin{tabular}{|l}
$\left.\mathrm{BEL}, \mathrm{r}_{\mathrm{Hob}}, \tau,+\right\rangle$ \\
$\mathbf{e}_{1}: \mathbf{p}_{\text {kill }} \mathbf{r}_{\text {witch }} \mathbf{r}_{\text {cow }}$ \\
$\mathbf{s}_{10}: \mathbf{p}_{\text {witch }} \mathbf{r}_{\text {witch }}$ \\
$\mathbf{s}_{11}: \mathbf{p}_{\text {cow }} \mathbf{r}_{\text {cow }} \mathbf{r}_{\text {Cob }}$
\end{tabular} \\
\hline $\begin{array}{l}\left\langle\mathrm{BEL}, \mathbf{r}_{\mathrm{Nob}}, \boldsymbol{\tau},+\right\rangle \\
\mathbf{e}_{\mathbf{2}}: \mathbf{p}_{\text {blight }} \mathbf{r}^{\prime \prime} \mathbf{r}_{\text {sow }} \\
\mathbf{s}_{\mathbf{2 1}}: \mathbf{p}_{\text {sow }} \mathbf{r}_{\text {sow }}{ }^{\prime} \mathbf{r}_{\text {Bob }}\end{array}$ \\
\hline
\end{tabular}


(c') Interpretation1:

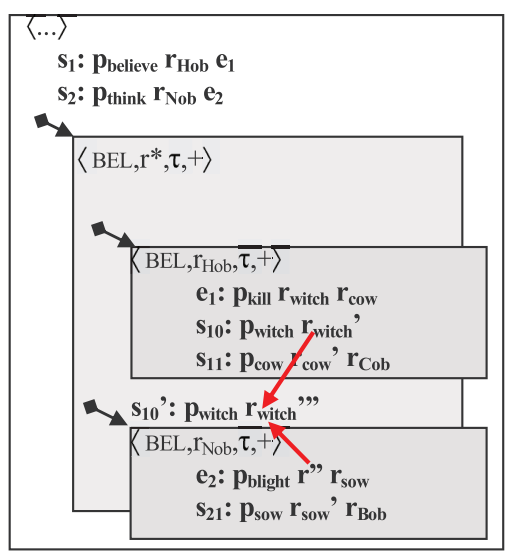

$\left(\mathrm{c}^{\prime \prime}\right)$ Interpretation2:

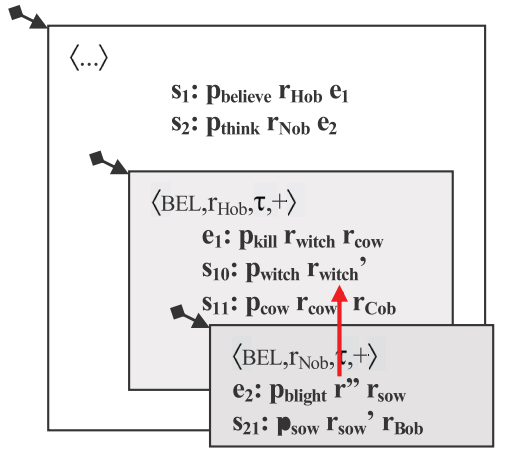

Thus we should have recourse to accommodation, again. What Zeevat (2005, 549) writes, serves as a confirmation: "Now the literature contains many explanations [...] Hob may have told Nob about his belief $\left[\rightarrow\left(10 \mathrm{c}^{\prime \prime}\right)\right]$, there may be a rumour in the village about a witch that has played a causal role in the formation of Hob's and Nob's beliefs $\left[\rightarrow\left(10 c^{\prime}\right)\right]$, there may have been an article in the local newspaper that Hob and Nob have each read $\left[\rightarrow\left(10 c^{\prime}\right)\right]$." Thus there might be different contexts "behind" this sentence, but the special use of she does require from the interpreter to consider some special contextual factor, that is, to accommodate some information concerning the context.

What is represented by the worldlet structure in $\left(10 c^{\prime}\right)$ in the framework of $\mathfrak{R e A L I S}$ would be paraphrased by Zeevat $(2005,540)$ as follows: Nob's relevant belief is over Hob's belief. In the other case (10c'), the two belief states are both over a certain "background" expressing the content of the rumor/the article; these two cases can be handled uniformly, by attributing the belief in the existence of a witch to a third person, $r^{*}$, who is the source of the rumor/author of the article. In the former case $\left(10 \mathrm{c}^{\prime \prime}\right)$, Hob's witch $r_{\text {witch }}^{\prime}$ has become directly accessible to Nob's referent $r^{\prime \prime}$ due to the embedding of the worldlets "floating" in the phase of $(10 \mathrm{~b})$ in the way triggered by the accommodated contextual information according to Zeevat's first scenario. In the latter case $\left(10 c^{\prime}\right)$, a new referent $r^{\prime \prime \prime}{ }_{\text {witch }}$ has been introduced which is accessible both to Hob's witch $r_{\text {witch }}^{\prime}$ and to Nob's referent $r$ " due to the embedding of the worldlets "floating" in 
the phase of (10b) in the way triggered by the accommodated contextual information according to Zeevat's second or third scenario. ${ }^{3}$

Now we are in a position to return to the problem of intersubjectivity, illustrated in (1d) in 1.1, because in our approach this problem is similar to the Hob-Nob problem.

We need the solution of this latter problem according to the scenario in $\left(10 c^{\prime}\right)$, where the speaker (i) is to attribute the referent of a witch $\left(r^{*}{ }_{\text {witch }}\right)$ to a (not necessarily known) "source" (of a rumor), denoted by $i^{*}$ in $\left(10 \mathrm{~d}^{\prime}\right)$ below, and this referent is situated in a way that both Hob's witch referent and Nob's witch referent will be "over" it in the sense used by Zeevat $(2005,540)$. Referent $r^{*}{ }_{\text {witch }}$, thus, is accessible to both referents in question (which are not accessible to each other directly). It is irrelevant, then, that there is no corresponding witch outside.

The figure in $\left(10 \mathrm{~d}^{\prime \prime}\right)$ below shows a similar configuration of referents, repeated twice.

(10) $\left(\mathrm{d}^{\prime}\right)$

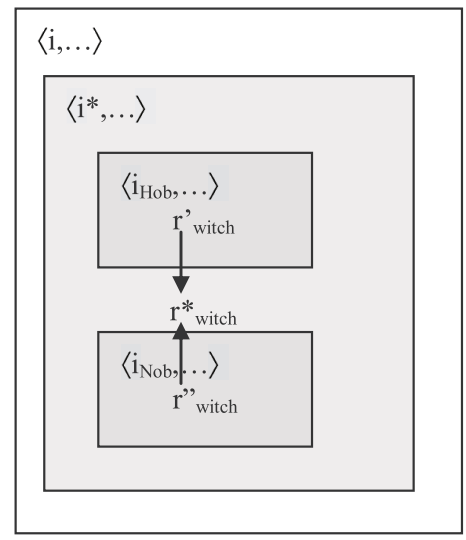

no witch

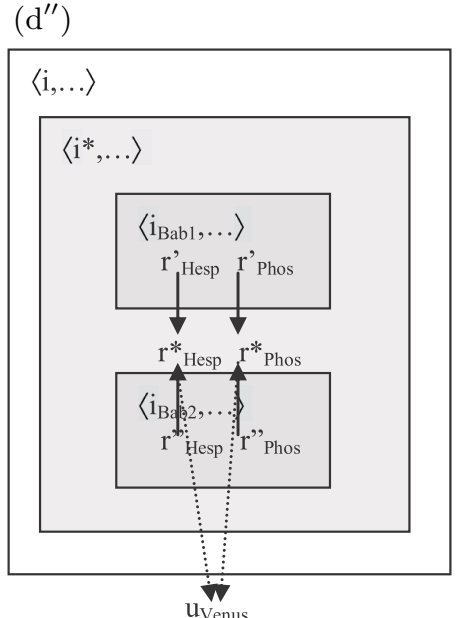

${ }^{3}$ One might think that the placement of Nob's worldlet of belief over Hob's $\left(10 \mathrm{c}^{\prime \prime}\right)$ or $r^{*}$ 's $\left(10 \mathrm{c}^{\prime}\right)$ worldlet of belief may result in the, incorrect, implication that Hob/ $r^{*}$ believes that Nob thinks that the witch who has blighted Bob's sow coincides with the witch who has killed Cob's cow. What is not (necessarily) true is that Hob/ $r^{*}$ knows about Nob's opinion at all. Fortunately, our representations in $\left(10 \mathrm{c}^{\prime} / \mathrm{c}^{\prime \prime}\right)$ do not contain a formula like this: s: $\mathbf{p b e l i e v e} \mathbf{r}_{\mathbf{H o b}} / \mathbf{r}^{*} \mathbf{s}_{\mathbf{2}}$; and as internal worlds are not closed under an operation which might produce this formula, our approach will not result in the incorrect implication in question. Nevertheless, in normal dialogues it can potentially be accommodated that if $i 2$ 's belief relies on $i 1$ 's belief, then $i 1$ is likely to realize $i 2$ 's belief. 
What corresponds to the rumor in Hob and Nob's village is the two Babylonians' assumed common background knowledge according to which there is a bright heavenly body seen in the evening sky, called Hesperus, and another star seen in the morning sky, called Phosphorus. What counts, is only the configuration of internal entities in the box structure expressing the relevant participants' beliefs; and that there is only one heavenly body outside instead of two entities is as irrelevant as the lack of a real witch outside in the Hob-Nob puzzle. The properly situated referents $r_{\text {Hesp }}^{*}$ and $r_{\text {Phos }}^{*}$, thus, have helped in accounting for the coreferentiality of the two Babylonians' two distinct "private objects", due to the fact that the former two referents are accessible to the latter four referents.

Landman's (1986) own solution is based on a mathematical construction that he calls "pegs": "they are the objects we assume in conversation" (128). In his opinion (131), "my view of the world represents my private belief and your view of the world yours, they still are information states, and if both ascribe properties to a peg, they do not each ascribe its own private properties to its own private peg, but they ascribe intersubjective properties to one and the same peg. And the same holds for the entities that figure in my belief about the world, and in my belief about your belief about the world. [...] While we were talking we may have reached a stage in which we could decide that two pegs were identical, because, relative to our assumed standard of precision, there were no facts left to be added to our information that could distinguish them." The internal entities serving as referents in ReALIS share certain properties with the partial objects that Landman calls pegs (see details in Alberti-Kleiber 2012), but we claim that it is a crucial innovation that our referents are regarded as belonging to mind representations consisting of worldlets that capture our beliefs, desires and intentions pertaining to both the external world and the content of each other's mind. It is through these peculiar representations that the above discussed referential chains can be set up, typically due to linguistic cues (e.g. pronouns, definite articles, proper names) and contextual circumstances, and almost never due to having reached, in a conversation, "a stage in which we could decide that two pegs were identical". It is not excluded at all that the two Babylonians in the example above know, think and believe radically different things about, say, Hesperus, while they can speak about it successfully. It is hopeless to calculate "the assumed standard of precision", mentioned above as a part of Landman's formulation, on the basis of which it should 
be decided whether two sets of pieces of information, i.e., "two partial objects", which may be very different, refer to the same thing in a given conversation, or not.

\section{Summary}

We have proposed a general solution to a related set of stubborn problems that the mainstream Kripke/Montague-inspired possible-worlds semantics suffers from: problems concerning expressions with the same reference/meaning/truth value, accessibility of referents, modal anchoring and intensional identity, and - generally-representing beliefs, desires, intentions.

This solution relies on embedding discourse representations - together with representations of interpreters' minds creating them- in the world model, by which we can get rid of the intermediate level of representation between linguistic form and world model, while preserving its content and relevant structural characteristics. In this way representationalist dynamic semanticists' useful referent hierarchies (visualized by Kampian boxes) are saved while antirepresentationalists' principal theoretical demand pertaining to the elimination of the "extra level" of representation has also been satisfied. Our approach, called ReALIS, also promises the reconciliation of the traditionally antipsychologist formal semantics and the cognitive approach whose representatives traditionally rejecting formalism. As for this latter topic, a second article (AlbertiKleiber 2012) will be devoted to the comparison of ReALIS with it and other seminal related works in the field: Landman's (1986) theory on partial objects, DRT (Kamp et al. 2011), SDRT (Asher-Lascarides 2003) and Situation Semantics. ${ }^{4}$

\section{References}

Alberti, Gábor 2000. Lifelong Discourse Representation Structures. In: Gothenburg Papers in Computational Linguistics 00-5 : 13-20.

Alberti, Gábor 2008. Paul D. Elbourne: Situations and individuals [review article]. In: Acta Linguistica Hungarica 55:427-39.

Alberti, Gábor 2009. ReALIS: An interpretation system which is reciprocal and lifelong. In: Workshop 'Focus on discourse and contex t-dependence' (16 Sept. 2009, Amsterdam Center for Language and Communication).

${ }^{4}$ A thorough comparison of $\Re$ ALIS with Situation Semantics has been published in Alberti's (2008) review article on Elbourne's (2005) book.

Acta Linguistica Hungarica 59, 2012 
Alberti, Gábor 2011a. ReALIS, avagy a szintaxis dekompozíciója [ReALIS, or the decomposition of syntax]. In: Általános Nyelvészeti Tanulmányok 23 :51-98.

Alberti, Gábor 2011b. ReALIS: interpretálók a világban, világok az interpretálóban [ReALIS: Interpreters in the world, worlds in the interpreter]. Akadémiai Kiadó, Budapest.

Alberti, Gábor - Judit Kleiber 2012. Where are possible worlds? II. Pegs, DRSs, worldlets and reification. In: Gábor Alberti - Judit Farkas - Judit Kleiber (eds): Vonzásban és változásban [In attraction and in change]. PTE Nyelvtudományi Doktori Iskola, Pécs.

Asher, Nicholas-Alex Lascarides 2003. Logics of conversation. Cambridge University Press, Cambridge.

Dekker, Paul 2000. Coreference and representationalism. In: Klaus von Heusinger - Urs Egli (eds): Reference and anaphoric relations, 287-310. Kluwer, Dordrecht.

Dowty, David R.-Robert E. Wall-Stanley Peters 1981. Introduction to Montague semantics. Reidel, Dordrecht.

Elbourne, Paul D. 2005. Situations and individuals. MIT Press, Cambridge MA.

Fagin, Ronald - Joseph Y. Halpern-Yoram Moses-Moshe Y. Vardi 1995. Reasoning about knowledge. MIT Press, Cambridge MA.

Farkas, Donka 1993. Modal anchoring and noun phrase scope. Ms. University of California at Santa Cruz.

Groenendijk, Jeroen-Martin Stokhof 1991. Dynamic predicate logic. In: Linguistics and Philosophy 14:39-100.

Kálmán, László 1990. Deferred information: The semantics of commitment. In: László Kálmán-László Pólos (eds): Papers from the 2nd Symposium on Logic and Language, 125-57. Akadémiai Kiadó, Budapest.

Kamp, Hans - Josef van Genabith - Uwe Reyle 2011. Discourse representation theory. In: Dov Gabbay - Franz Guenthner (eds): Handbook of philosophical logic, Vol. 15: 125-394. Springer, Berlin.

Kiefer, Ferenc 2000. Jelentéselmélet [Semantic theory]. Corvina, Budapest.

Landman, Fred 1986. Towards a theory of information. Foris, Dordrecht.

Pollard, Carl 2007. Hyperintensions. ESSLLI 2007, http://www.cs.tcd.ie/esslli2007.

Roberts, Craige 1996a. Anaphora in intensional contexts. In: Shalom Lappin (ed.): The handbook of contemporary semantic theory, 215-46. Blackwell, Cambridge MA \& Oxford.

Roberts, Craige 1996b. Information structure in discourse: Towards an integrated formal theory of pragmatics. In: Jae Hak Yoon-Andreas Kathol (eds): OSU Working Papers in Linguistics 49: Papers in Semantics, 91-136. The Ohio State University, Columbus.

Schnell, Zsuzsanna 2006. Metafora és metareprezentáció - egy mentalista modell [Metaphor and metarepresentation: A mentalistic model]. In: Világosság 47:111-29. (http://www.epa.hu/01200/01273/00034/pdf/20070507213650.pdf)

Zeevat, Henk 2005. Overlaying contexts of interpretation. In: Emar Maier-Corien Bary - Janneke Huitink (eds): Proceedings of SuB9. (http://www.ru.nl/ncs/sub9) 